\title{
MULTISCALE GUIDED DEBLURRING: CHROMATIC ABERRATION CORRECTION IN COLOR AND NEAR-INFRARED IMAGING
}

\author{
Zahra Sadeghipoor ${ }^{1}$, Yue M. Lu ${ }^{2}$, Erick Mendez ${ }^{3}$, and Sabine Süsstrunk ${ }^{1}$ \\ ${ }^{1}$ School of Computer and Communication Sciences, EPFL, Lausanne, Switzerland \\ ${ }^{2}$ Harvard School of Engineering and Applied Sciences, Cambridge, MA 02138, USA \\ ${ }^{3}$ Qualcomm Inc., Vienna, Austria
}

\begin{abstract}
Chromatic aberration, caused by photographic lens imperfections, results in the image of only one spectral channel being sharp, while the other channels are blurred depending on their wavelengths difference with the sharp channel.

We study chromatic aberration for a system that jointly records color and near-infrared (NIR) images on a single sensor. Chromatic aberration in such a system leads to a blurred NIR image when the color image is in-focus and sharp. We propose an algorithm that deblurs the NIR image using the gradients of the sharp color image, as both scene representations are generally similar. However, the details of these images often exhibit significant differences due to varying scene reflection and absorption in the corresponding bands. To account for this, we compute the correlation between color and NIR gradients, and use the gradients of the color image in reconstructing NIR only where the gradients are highly correlated. We propose a multiscale scheme that gradually deblurs NIR and accurately computes similarities between color and NIR gradients. Experimental results show that our algorithm recovers details of NIR without producing visible artifacts.
\end{abstract}

Index Terms - Axial chromatic aberration, NIR imaging, similarity maps, gradient-based deblurring.

\section{INTRODUCTION}

The refractive index of a photographic lens depends on the wavelength of the incoming light. Hence, light rays with different wavelengths converge at different distances. If the sensor is placed at the focus plane of one wavelength, the image of only that wavelength is sharp, while light rays with different wavelengths produce blurred images (Fig. 1-a). This phenomenon is called axial chromatic aberration.

Any multispectral camera that uses the same optical path to capture all spectral channels suffers from axial chromatic aberration. We study this phenomenon in a system that simultaneously captures color (red, green, blue) and near-infrared (NIR) channels. NIR is part of the electromagnetic spectrum with a wavelengths range of approximately $700-1100 \mathrm{~nm}$, adjacent to the visible spectrum. Fig. 1-(b) shows a pair of color
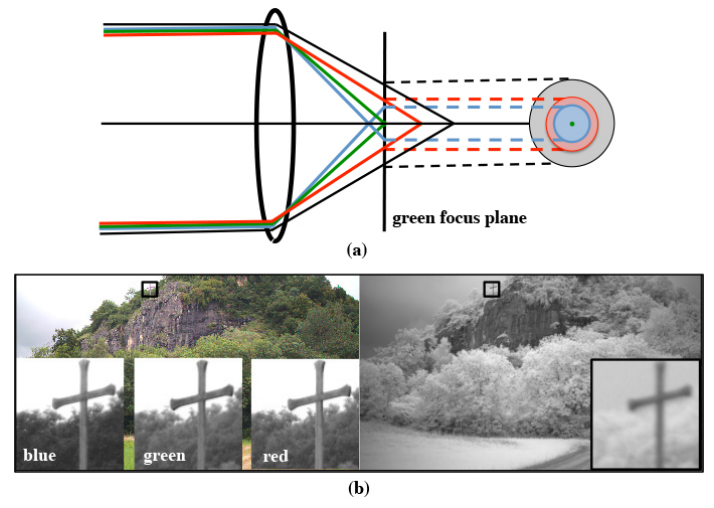

Fig. 1. (a) A simple lens converges light rays with different wavelengths at different distances. If the sensor is at the focus plane of green, only the image of radiation in this wavelength is sharp. The disks illustrate the blur kernels for other wavelengths (black rays: NIR radiation). (b) The color image is in-focus and all color channels are sharp. The NIR image captured with the same lens and focus is blurred. The differences are most noticeable while viewed on a screen.

and NIR images representing the same scene.

The growing interest in joint capture of color and NIR images on a single sensor [1-4] is caused by recent developments in using these images in computational photography and computer vision. Combining color and NIR has proven useful in low light imaging [5], scene recognition [6], shadow detection [7], etc. These applications are enabled as siliconsensors, used in most digital cameras, are sensitive to both visible and NIR radiations. Hence, the joint acquisition of color and NIR images on a single sensor is feasible.

Compound lenses that correct CA for both visible and NIR bands are usually too bulky and costly to be used with consumer cameras. Hence, our aim is to use a simpler lens and to correct for chromatic aberration by post-processing NIR and color images. We consider the scenario where the color image is in-focus and sharp, and NIR is out-of-focus and blurred, and the task of reducing CA distortions is equivalent to deblurring the NIR image. Figure 1-(b) shows color 
and NIR images captured with the same lens and same focus. The lens, corrected for visible wavelengths, results in sharp color channels, while the NIR channel is blurred.

In our problem, the sharp color image representing the scene is available and can be used to deblur NIR assuming that the edges of color and NIR images are strongly correlated. This assumption holds for many, but not all regions in the image. Differences of light and surface reflections in visible and NIR bands result in intrinsic variations between the edges of these images (see Fig. 2), and simply adding the high-frequency details of the color image to NIR discards these differences. This causes many of the algorithms that fuse color and NIR and depend on the differences between these images to fail.

To preserve the inherent differences between color and NIR, we use the gradients of the color image in deblurring NIR only at pixels where the gradients of these images are similar. We propose a multiscale algorithm that accurately estimates the similarity values between color and NIR gradients at each pixel. We call the matrix that contains the similarity values the "similarity map". Our method iterates between deblurring NIR and computing similarity maps. As the maps at each scale are formed based on a deblurred NIR image, they provide an accurate estimation of the correlation between color and NIR gradients.

To correct chromatic aberration in color imaging, it is often assumed that the edges of color channels are strongly correlated. Following this assumption, the high-frequency information of the sharp channel is used to retrieve the lost details in the images of other channels [8-11]. As the high-frequency components of color and NIR images are not always correlated, this approach is prone to failure in our scenario.

In [12], we proposed a guided deblurring algorithm that maintains the differences between color and NIR by roughly estimating the similarity values from the gradients of color and blurred NIR images. The gradients of the blurred NIR significantly differ from the underlying sharp image. Hence, using the blurred image results in low-accuracy estimation of similarities degrading the performance of a guided deblurring algorithm. In this paper, we propose a multiscale deblurring approach that ensures the similarity values are estimated accurately from a deblurred NIR image. In Section 3, we show

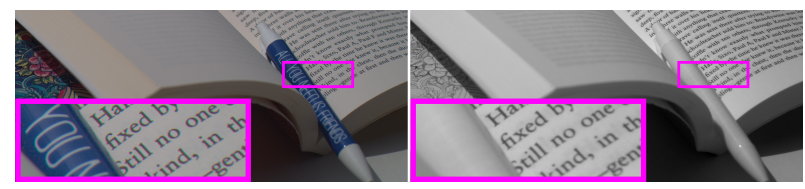

Fig. 2. The color pigments on the pen are transparent in NIR, resulting in the gradients of color and NIR images being different. The text in the book looks the same, as carbon black used in modern ink absorbs light in both visible and NIR. that the proposed multiscale algorithm outperforms [12].

\section{MULTISCALE GUIDED DEBLURRING}

We mathematically formulate blurring of the NIR image as:

$$
\mathcal{N}_{b}=k * \mathcal{N}
$$

where $\mathcal{N}$ is the ideal sharp NIR, $\mathcal{N}_{b}$ is the blurred NIR image, $k$ is the lens blur kernel, and $*$ stands for convolution.

Our algorithm is guided by a gray-scale representation of the color image, which contains the spatial information about the scene. We call this representation $Y$ and compute it as the average of the three color channels.

We form Gaussian pyramids of $Y$ and $\mathcal{N}_{b}$ (blurred NIR) images with $p+1$ scales. In each scale, the images are downsampled by a factor $R$ to form the images of the next coarser level. The full-resolution images are called $\mathcal{N}_{b}^{(0)}$ and $Y^{(0)}$, and the coarsest scale is denoted by $\mathcal{N}_{b}^{(p)}$ and $Y^{(p)}$. We represent downsampling and upsampling by factor $R$, respectively, as $(.)_{\downarrow R}$ and $(.)_{\uparrow R}$. Fig. 3 demonstrates the notations.

Assuming that the lens blur kernel is modeled as a Gaussian filter $[13,14]$, we estimate the kernel in the finest scale of the pyramid by solving the following optimization problem and computing the variance of the Gaussian filter:

$$
\begin{aligned}
& k^{(0)}=f\left(\mathcal{N}_{b}^{(0)}, Y^{(0)}\right) \triangleq \underset{k}{\operatorname{argmin}}\left\|\nabla \mathcal{N}_{b}^{(0)}-k * \nabla Y^{(0)}\right\|_{F}^{2} \\
& \text { s.t. } k(m, n)=\frac{1}{c} \exp \left(-\frac{m^{2}+n^{2}}{2 \sigma^{2}}\right) .
\end{aligned}
$$

$(m, n)$ are horizontal and vertical coordinates, and $\sigma$ is the standard deviation of the Gaussian filter (the blur kernel). $c$ is a scalar ensuring that the sum of kernel elements is 1 . This optimization is based on the assumption that in many regions of the image, color and NIR gradients are correlated.

We start the blur kernel estimation from the finest scale of the pyramid as the full-resolution $Y$ and NIR images exhibit larger differences compared with the downsampled image pairs. After estimating $k^{(0)}$, the blur kernels in coarser levels are sequentially computed by downsampling the kernel in the previous finer scale by the factor $R$, i.e.,

$$
k^{(1)}=\left(k^{(0)}\right)_{\downarrow R}, \quad k^{(2)}=\left(k^{(1)}\right)_{\downarrow R}, \cdots .
$$

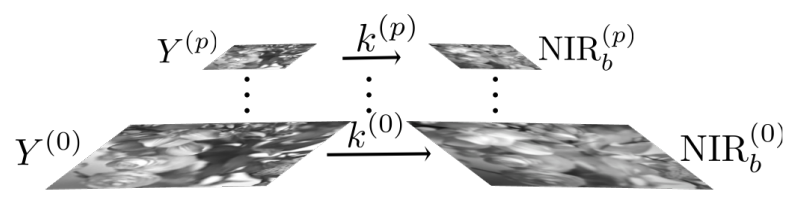

Fig. 3. A pyramid of NIR and $Y$ images with $p+1$ scales. The kernel in each scale is obtained by downsampling the kernel in the previous finer scale. 


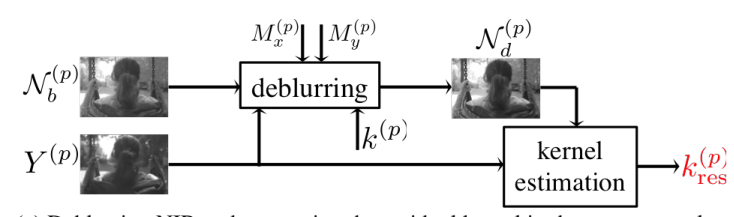

(a) Deblurring NIR and computing the residual kernel in the coarsest scale.

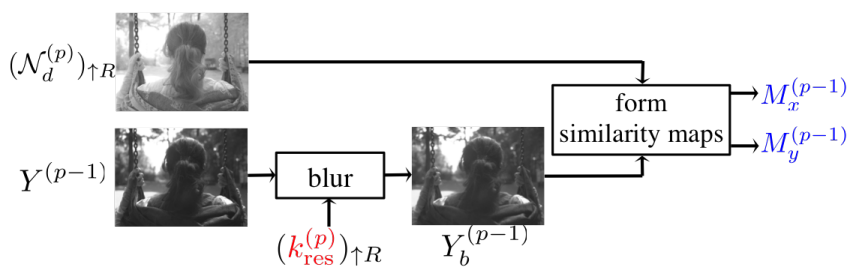

(b) Forming similarity maps using the NIR image deblurred in the previous scale.

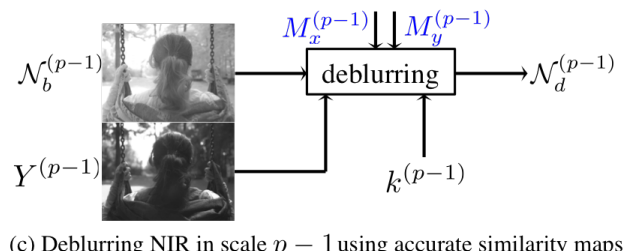

Fig. 4. A schematic of our multiscale deblurring algorithm for scales $p$ and $p-1$. The procedure shown in (a) and (b) is repeated until the full-resolution NIR is deblurred.

We first deblur the NIR image in the coarsest scale of the pyramid by solving the following problem:

$$
\begin{aligned}
\mathcal{N}_{d}^{(p)} & =g\left(\mathcal{N}_{b}^{(p)}, Y^{(p)}, M_{x}^{(p)}, M_{y}^{(p)}\right) \\
& \triangleq \underset{\mathcal{N}^{(p)}}{\operatorname{argmin}} \lambda\left\|\mathcal{N}_{b}^{(p)}-k^{(p)} * \mathcal{N}^{(p)}\right\|_{F}^{2} \\
& +\sum_{l \in\{x, y\}}\left\|\nabla_{l} \mathcal{N}^{(p)}-M_{l}^{(p)} \odot \nabla_{l} Y^{(p)}\right\|_{F}^{2}
\end{aligned}
$$

where $\nabla_{l}$ represents horizontal and vertical gradient operators, $\odot$ denotes the element-wise multiplication, and $\lambda$ is the regularization parameter. $M_{l}^{(p)}$ for $l \in\{x, y\}$ are horizontal and vertical similarity maps in scale $p$.

Incorporating the similarity maps in the second term of (4) ensures that the gradients of $Y^{(p)}$ are exploited in reconstructing the sharp NIR image only where the maps have high values. In those pixels the edges of NIR and Y channels are strongly correlated.

Only for the coarsest scale of the pyramid, we compute the similarity maps, similar to [12], by comparing the gradients of blurred NIR and blurred $Y$ images:

$M_{l}^{(p)}(m, n)=1-\frac{\left|\nabla_{l} \mathcal{N}_{b}^{(p)}(m, n)-\nabla_{l} Y_{b}^{(p)}(m, n)\right|}{\left|\nabla_{l} \mathcal{N}_{b}^{(p)}(m, n)+\nabla_{l} Y_{b}^{(p)}(m, n)\right|}, l \in\{x, y\}$

Here $Y_{b}^{(p)}=k^{(p)} * Y^{(p)}$, and $(m, n)$ indicates the pixel at row $m$ and column $n$.
If we use the original sharp $Y$ image in (5), the similarity values would be low even where $Y$ and NIR gradients are inherently similar, as all NIR edges are changed by blur. However, the similar edges would have approximately the same profile when both images ( $Y$ and NIR) are blurred. Figure 4(a) shows deblurring NIR in the coarsest scale of the pyramid.

The gradients of blurred NIR and blurred $Y$ images in (5) provide a rough approximation of similarities between sharp color and NIR images. Hence, the output image of (4), $\mathcal{N}_{d}^{(p)}$, is still blurred compared with the underlying sharp NIR image, $\mathcal{N}^{(p)}$. The residual blur in $\mathcal{N}_{d}^{(p)}$ can be formulated as:

$$
\mathcal{N}_{d}^{(p)}=k_{\mathrm{res}}^{(p)} * \mathcal{N}^{(p)} .
$$

We estimate the residual kernel, $k_{\text {res }}^{(p)}$, by solving:

$$
k_{\text {res }}^{(p)}=f\left(\mathcal{N}_{d}^{(p)}, Y^{(p)}\right) .
$$

$f(.,$.$) is defined in (2). See Fig. 4-(a) for an illustration.$

To deblur the NIR image in the next scale, $\mathcal{N}_{b}^{(p-1)}$, we need to compute the corresponding similarity maps $\left(M_{x}^{(p-1)}\right.$, $\left.M_{y}^{(p-1)}\right)$. If, similar to the previous scale, we use the blurred NIR image, inaccurate estimations of true similarity maps are obtained. Instead, we propose to use an upsampled version of the image deblurred in the previous scale, $\left(\mathcal{N}_{d}^{(p)}\right)_{\uparrow R}$. This image is more similar to the ideal sharp NIR image at scale $p-1$, and provides more accurate estimations of similarity maps. Thus, we compute $M_{x}^{(p-1)}$ and $M_{y}^{(p-1)}$ as follows:

$$
M_{l}^{(p-1)}=1-\frac{\left|\nabla_{l}\left(\mathcal{N}_{d}^{(p)}\right)_{\uparrow R}-\nabla_{l} Y_{b}^{(p-1)}\right|}{\left|\nabla_{l}\left(\mathcal{N}_{d}^{(p)}\right)_{\uparrow R}+\nabla_{l} Y_{b}^{(p-1)}\right|}, l \in\{x, y\},
$$

Note that $\mathcal{N}_{d}^{(p)}$ after upsampling by factor $R$ and $Y_{b}^{(p-1)}$ have the same resolution.

Similar to the previous scale, $Y_{b}^{(p-1)}$ in (8) is the $Y$ image deblurred by the residual blur kernel of $\left(\mathcal{N}_{d}^{(p)}\right)_{\uparrow R}$. To compute this blur kernel, we upsample $k_{\text {res }}^{(p)}$ in (7) by factor $R$. So:

$$
Y_{b}^{(p-1)}=\left(k_{\mathrm{res}}^{(p)}\right) \uparrow R * Y^{(p-1)} .
$$

Figure 4-(b) shows the process of estimating similarity maps.

The similarity maps computed in (8) are then used in the following optimization problem to deblur $\mathcal{N}_{b}^{(p-1)}$ (Fig. 4-c):

$$
\mathcal{N}_{d}^{(p-1)}=g\left(\mathcal{N}_{b}^{(p-1)}, Y^{(p-1)}, M_{x}^{(p-1)}, M_{y}^{(p-1)}\right),
$$

where function $g(., ., .,$.$) is defined in (4).$

The deblurring algorithm is applied to every scale of the pyramid until $\mathcal{N}_{b}^{(0)}$ (the full-resolution NIR) is deblurred to obtain $\mathcal{N}_{d}^{(0)}$, which is the final output of the algorithm. 


\section{EXPERIMENTAL RESULTS}

We captured the images used in these experiments by a Canon Rebel T1i camera, after removing its NIR-blocking filter. Such a camera is sensitive to both visible and NIR radiations. For each scene, we sequentially captured one color image by placing an NIR-blocking filter in front of the lens and two NIR images using a visible-light blocking filter. The color image is in focus, and the first NIR image captured with the same focus is blurred. We then refocused the camera to capture a sharp NIR image, used as the ground-truth. We align all three images for each scene using feature-point matching. Note that the ideal color and NIR camera captures the images in one shot, and it is not possible to set the focus differently for color and NIR. In our experiments, we created this situation by not changing the focus settings from color to the NIR shot. We use the downsampling factor of $R=4$.

Table 1 reports the PSNR values averaged over $20 \mathrm{im}$ ages deblurred by our method, the algorithm of [12], the blind deblurring algorithm of [15], and the guided filtering algorithm [16]. We applied the guided filtering algorithm to deblur the NIR image using the sharp color image as a guide. Table 1 shows that our method outperforms other algorithms. The following visual comparisons demonstrate the advantage of our method more clearly.

Figure 5 compares the images deblurred by the algorithm of [12] and our multiscale deblurring where $p=2$ (an image pyramid with three scales) is used. It is immediately observed that more accurate similarity maps computed by our multiscale algorithm help recovering sharper details. At the same time, our algorithm still successfully preserves the inherent differences between color and NIR images.

In Fig. 6-(c), we present the results of simply adding the high-frequency details of the color image into the blurred NIR image at every pixel. This approach is similar to the chromatic aberration correction algorithms that assume strong correlation between the gradients of all color channels [10]. To obtain the results in Fig. 6-(c), we solve the following problem:

$$
\mathcal{N}_{d}=\operatorname{argmin}\left\|\mathcal{N}_{b}-k * \mathcal{N}\right\|_{F}^{2}+\sum_{l \in\{x, y\}}\left\|\nabla_{l} \mathcal{N}-\nabla_{l} Y\right\|_{F}^{2} .
$$

This method cannot preserve the differences between color and NIR images. For instance, in Fig. 6, the text on the pen is almost invisible in the ground-truth NIR, however, the image deblurred by (11) contains the text (the first row of the figure).

We present the results of the guided image filtering [16] in Fig. 6-(d). The deblurred NIR image contains false edges

\begin{tabular}{|c||c|c|c|c|}
\hline & $\begin{array}{c}\text { method of } \\
{[15]}\end{array}$ & $\begin{array}{c}\text { guided filtering } \\
{[16]}\end{array}$ & $\begin{array}{c}\text { method of } \\
{[12]}\end{array}$ & Ours \\
\hline PSNR & 27.58 & 28.10 & 29.65 & 30.22 \\
\hline
\end{tabular}

Table 1. The PSNR values averaged over 20 deblurred images.

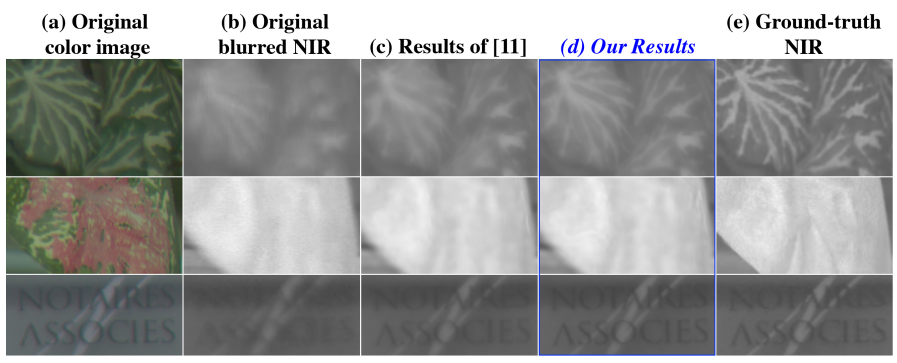

Fig. 5. Crops of (a) sharp color and (b) blurred NIR images captured with the same focus settings. (c) and (d) compare the performance of our previous algorithm [12] and our multiscale deblurring method.

and even the pixel intensities are not similar to the groundtruth. Our algorithm produces sharp images while preserving the inherent differences between color and NIR images.

\section{CONCLUSION}

We study chromatic aberration in the joint acquisition of color and NIR images. If the camera is focused while capturing the color image, the NIR image recorded with the same focus is blurred. We propose a multiscale algorithm that effectively uses the gradients of the color image to deblur NIR and preserves the inherent differences between these images. To achieve this, we estimate accurate similarities between color and NIR gradients. Our multiscale technique provides such accurate estimations by iterating between deblurring NIR and updating the similarity values. Our algorithm outperforms the guided image filtering [16], the deblurring method of [15], and our previous method [12].

In this study, we considered the case where the NIR image is uniformly blurred. However, the amount of lens blur depends on the object depth. Addressing spatially varying blur kernels is part of our future research.

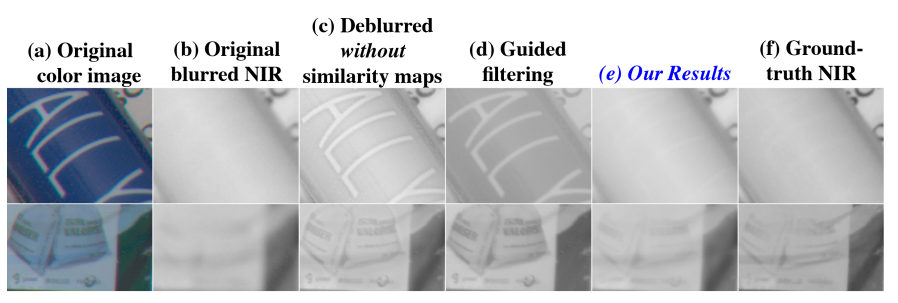

Fig. 6. (c) Deblurring without using similarity maps introduces false edges that are not present in the ground-truth NIR. (d) The guided image filtering [16] does not preserve the differences between NIR and color. The pen is dark in the first row of (d), while it is bright in the ground-truth NIR image. (e) Our algorithm maintains the intrinsic differences between color and NIR. 


\section{REFERENCES}

[1] Y. M. Lu, C. Fredembach, M. Vetterli, and S. Süsstrunk, "Designing color filter arrays for the joint capture of visible and near-infrared images," in IEEE International Conference on Image Processing (ICIP), 2009.

[2] G. Langfelder, T. Malzbender, A. F. Longoni, and F. Zaraga, "A device and an algorithm for the separation of visible and near infrared signals in a monolithic silicon sensor," in SPIE Proceedings: Visual Information Processing and Communication II, 2011, vol. 7882.

[3] Z. Sadeghipoor, Y. Lu, and S. Süsstrunk, "A novel compressive sensing approach to simultaneously acquire color and near-infrared images on a single sensor," in IEEE International Conference on Acoustics, Speech, and Signal Processing (ICASSP), 2013.

[4] X. Y. Luo, J. Zhang, and Q. H. Dai, "Hybrid fusion and demosaicing algorithm with near-infrared image," in SPIE Proceedings: Multisensor, Multisource Information Fusion: Architectures, Algorithms, and Applications, 2014, vol. 9121.

[5] D. Krishnan and R. Fergus, "Dark flash photography," in ACM SIGGRAPH, 2009.

[6] M. Brown and S. Süsstrunk, "Multispectral SIFT for scene category recognition," in IEEE Conference on Computer Vision and Pattern Recognition (CVPR), 2011.

[7] D. Rüfenacht, C. Fredembach, and S. Süsstrunk, “Automatic and accurate shadow detection using nearinfrared information," IEEE Transactions on Pattern Analysis and Machine Intelligence (TPAMI), vol. 36, pp. 1672-1678, 2014.

[8] S. B. Kang, "Automatic removal of chromatic aberration from a single image," in IEEE Conference on Com- puter Vision and Pattern Recognition (CVPR), 2007.

[9] S. W. Chung, B. K. KIM, and W. J. Song, "Detecting and eliminating chromatic aberration in digital images," in IEEE International Conference on Image Processing (ICIP), 2009.

[10] C. Tisse, H. P. Nguyen, R. Tessiéres, M. Pyanet, and F. Guichard, "Extended depth-of-field (EDoF) using sharpness transport across colour channels," in SPIE Proceedings: Novel Optical Systems Design and Optimization XI, 2008.

[11] F. Heide, M. Rouf, M. B. Hullin, B. Labitzke, W. Heidrich, and A. Kolb, "High-quality computational imaging through simple lenses," ACM Transactions on Graphics (TOG), vol. 32, 2013.

[12] Z. Sadeghipoor, Y. M. Lu, and S. Süsstrunk, "Gradientbased correction of chromatic aberration in the joint acquisition of color and near-infrared images," in Proc. of IS\&T/SPIE Electronic Imaging: Digital Photography and Mobile Imaging XI, 2015, vol. 9404.

[13] B. Tatian, "Method for obtaining the transfer function from the edge response function," Journal of the Optical Society of America, vol. 55, pp. 1014-1019, 1965.

[14] S. Hwang, K. Kim, J. Shin, J. Paik, B. Abidi, and M. Abidi, "Real-time digital autofocusing using a priori estimated set of PSFs," in SPIE Proceedings: RealTime Imaging VIII, 2004, vol. 5297.

[15] D. Krishnan, T. Tay, and R. Fergus, "Blind deconvolution using a normalized sparsity measure," in IEEE Conference on Computer Vision and Pattern Recognition (CVPR), 2011

[16] K. He, J. Sun, and X. Tang, "Guided image filtering," IEEE Transactions on Pattern Analysis and Machine Intelligence (TPAMI), vol. 35, pp. 1397-1409, 2013. 